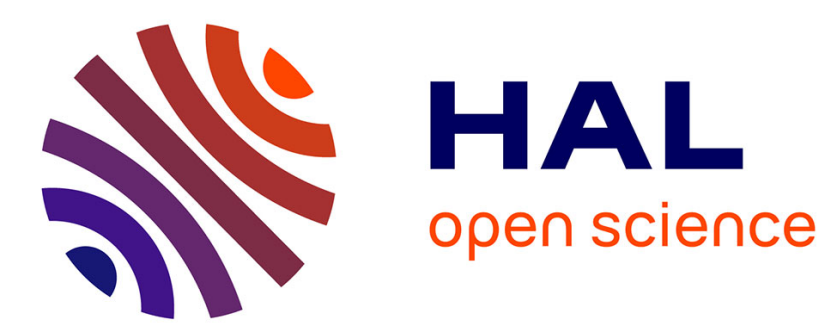

\title{
Le cartulaire de la seigneurie de Germiny réalisé en 1540 pour Nicolas de Lutzelbourg
}

Jean-Christophe Blanchard

\section{To cite this version:}

Jean-Christophe Blanchard. Le cartulaire de la seigneurie de Germiny réalisé en 1540 pour Nicolas de Lutzelbourg. 2010. halshs-01264567

\section{HAL Id: halshs-01264567 \\ https://shs.hal.science/halshs-01264567}

Preprint submitted on 29 Jan 2016

HAL is a multi-disciplinary open access archive for the deposit and dissemination of scientific research documents, whether they are published or not. The documents may come from teaching and research institutions in France or abroad, or from public or private research centers.
L'archive ouverte pluridisciplinaire HAL, est destinée au dépôt et à la diffusion de documents scientifiques de niveau recherche, publiés ou non, émanant des établissements d'enseignement et de recherche français ou étrangers, des laboratoires publics ou privés. 
Jean-Christophe BLANCHARD

Centre de Médiévistique Jean Schneider

ERL 7229 Université de Lorraine-CNRS

\section{Le cartulaire de la seigneurie de Germiny réalisé en 1540 pour Nicolas de Lutzelbourg}

\section{Introduction :}

Nicolas de Lutzelbourg (v. 1485-1547) est d'abord connu comme le maître d'ouvrage d'un château construit à Fléville dans les années 1530. Sa réalisation fut confiée à un architecte originaire de Saint-Mihiel, Michel de La Chausse, qui, intégrant le donjon du XIV siècle, conçut un ensemble Renaissance de fort belle facture ${ }^{1}$. Le seigneur de Fléville fut aussi, mais cela est plus confidentiel, le commanditaire et propriétaire d'un armorial conservé à la Bibliothèque municipale de Nancy $^{2}$. Pourtant ce beau manuscrit révèle autant que le château, les goûts fastueux du personnage. La fortune qui rend possible cette magnificence est due au service du prince et à sa rétribution mais elle est surtout le produit de différentes seigneuries et de leur gestion attentive.

Un cartulaire composé en 1540 témoigne de ce souci. Il concerne les terres de Germiny, Mazirot et Offroicourt ${ }^{3}$, dont Nicolas est devenu seigneur par héritage maternel. Ce cartulaire de seigneurie ${ }^{4}$, non mentionné par Georges Poull dans son étude sur le château et les seigneurs de Fléville ${ }^{5}$, absent du répertoire des cartulaires français laïques établi par Lucie Fossier et Olivier Guyotjeannin ${ }^{6}$, est quasi inconnu. C'est pourtant un document extrêmement précieux tant sur le plan de l'histoire d'une seigneurie et de sa gestion, que sur le plan de l'étude des cartulaires.

\section{Histoire du document :}

L'histoire du cartulaire de Nicolas de Lutzelbourg peut aisément se retracer puisqu'il est encore de nos jours conservé dans les collections du duc de Mouchy, descendant en ligne collatérale du commanditaire.

\section{Nicolas de Lutzelbourg :}

Mais avant d'analyser le cartulaire et son contenu, il convient de donner quelques précisions biographiques concernant Nicolas de Lutzelbourg pour mieux le situer dans la société de son temps. Issus d'une famille seigneuriale originaire du village éponyme de la vallée supérieure de la Sarre, les Lutzelbourg, vassaux des évêques de Metz, s'implantèrent dans le duché de Lorraine par le mariage de Jean de Lutzelbourg avec Ide, fille de Jean de

\footnotetext{
${ }^{1}$ Cécile BertRAND, «Le château de Fléville », Congrès Archéologique de France, $164{ }^{\mathrm{e}}$ session, 2006, Nancy et Lorraine méridionale, Paris, 2008, p. 29-37.

${ }^{2}$ Ms. 1727. Sur ce manuscrit : Jean-Christophe BLANCHARD, «L'Armorial de Jean de Haraucourt, manuscrit du $\mathrm{XVI}^{\mathrm{e}}$ siècle conservé au Musée lorrain, à Nancy », Lotharingia, X, 2001, p. 81-125, pour l'armorial de Lutzelbourg : p. 83 et 86.

${ }^{3}$ Meurthe-et-Moselle, ar. Toul, c. Colombey-les-Belles ; Vosges, ar. Neufchâteau, c. Mirecourt et id., c. Vittel. Une analyse des actes contenus dans le cartulaire est donnée en annexe.

${ }^{4}$ Lucie FOSSIER et Olivier GUYOTJEANNIN, «Cartulaires français laïques : seigneuries et particuliers », Les cartulaires. Actes de la Table ronde organisée par l'Ecole nationale des chartes et le G.D.R. 121 du C.N.R.S. (Paris, 5-7 décembre 1991), Paris, 1993, p. 379-410, ici p. 380-382.

${ }^{5}$ Georges Poull, Fléville. Son château et ses seigneurs XIII ${ }^{e}$ s.-XIX ${ }^{e}$ s. Histoire détaillée de cette demeure et de ses possesseurs, les Fléville, les Lutzelbourg et les Beauvau, Rupt-sur-Moselle, 1988.

${ }^{6}$ Lucie FossiER et Olivier GUYOTJEANNIN, op. cit., pour les répertoires p. 398-408.
} 
Fléville dit le Moine ${ }^{7}$. Leur fils Eguenolf de Lutzelbourg hérita des biens de son oncle Warry de Fléville après son décès en 1466. De son mariage avec Elisabeth de Harange, Eguenolf eut plusieurs enfants : Bernard, lieutenant du marquis de Bade à Luxembourg, Henri, seigneur de Lutzelbourg, et Warry, seigneur de Fléville ${ }^{8}$. Ce dernier épousa en 1482 Béatrix de Germiny, veuve de Philibert du Châtelet, qui lui apporta Geminy, Mazirot et Offroicourt (Fig. 1) Nicolas de Lutzelbourg est né de cette union vers 1485. Ce dernier, dont la mère mourut en 1495, a d'abord été seigneur de Mazirot. Il prit part aux campagnes d'Italie et était présent aux côtés du duc Antoine à Agnadel le 14 mai 1509. Il ne devint conseiller du duc puis capitaine d'Epinal qu'en 1523. Peu après, en 1525, il participa à la Guerre des Rustauds. Dans les mêmes moments, il avait épousé Marguerite de Lucy, fille de Mathieu de Lucy et de Claude de Craincourt. Nicolas devint seigneur de Fléville à la mort de son père en 1528. Avant 1532, il fut remplacé à l'office de capitaine d'Epinal par Jacquot de Savigny. La reconstruction du château de Fléville commença en 1533. C'est sans doute dans les années 1530-1540 qu'il fit enluminer son armorial. Le cartulaire fut quant à lui écrit en 1540. Le seigneur de Fléville fut encore nommé capitaine de Nancy en $1543^{10}$. Homme d'expérience, fidèle de la Maison ducale de Lorraine, Nicolas fut choisi comme maître temporel des cérémonies qui ont été célébrées à Nancy en juin 1546 lors de la pompe funèbre du duc Antoine $(\dagger 1544)^{11}$. Ayant sans doute pris conscience à cette occasion que «toute humaine nature est subgecte au dangier inevitable de la mort », il dicta son testament le 9 août de cette même année. Il décèda le 5 août 1547, ne laissant aucun héritier mâle, et fut inhumé dans la chapelle Saint-Nicolas jadis fondée par les Fléville en l'église abbatiale de Clairlieu ${ }^{12}$.

\section{Après la mort de Nicolas :}

Sa veuve lui survécut jusqu'en 1555 ; leur succession ne fut réglée qu'en 1563 à l'issu d'un long débat. Les biens furent alors enfin partagées entre les cinq descendantes du couple ou leurs ayants droit : Louise et Jean de Haraucourt, Renée et Nicolas de Choiseul, Barbe et Jean de Ludres, Claude de Beauvau, veuf de Nicole, au nom de ses enfants, et Anne, veuve de Robert de Boullant ${ }^{13}$. On constate que tous emportèrent une part de Fléville et de Germiny. Mais il n'en alla pas de même de Mazirot et d'Offroicourt. La première seigneurie fut partagée en deux, une moitié revint à Renée et Nicolas de Choiseul, l'autre à Anne de Lutzelbourg. La seconde fut également répartie par moitié entre Louise et Jean de Haraucourt et Barbe et Jean de Ludres. Le cartulaire passa alors aux mains de Claude de Beauvau et resta dans cette famille jusqu'au mariage d'Anne Louise de Beauvau avec Louis Philippe Marc Antoine de Noailles, duc de Mouchy, en 1767(Fig. 2) ${ }^{14}$. Rien pourtant ne prédisposait Claude à devenir propriétaire de ce cartulaire. Il n'avait pas plus de droit que les autres co-héritiers de Fléville sur ce document. Peut-être même moins puisqu'il n'avait rien reçu de Mazirot et d'Offroicourt. Mais son lot comprenait la «grant tour ancienne du grand chasteau de Fléville », la tour quadrangulaire de l'angle sud-ouest du château actuel. Au rez-de-chaussée

\footnotetext{
${ }^{7}$ Moselle, ar. Sarrebourg, c. Phalsbourg ; Fritz EYER, «Les familles nobles de Lutzelbourg », Société d'histoire et d'archéologie de Saverne et environs. Cahiers trimestriels «Pays d'alsace», t. 82-83, 1973, p. 29-38; Georges Poull, op. cit., p. 26.

${ }^{8}$ Georges Poull, op. cit., p. 35, 38.

${ }^{9}$ Cf. en annexe le contenu du cartulaire : ${ }^{\circ}$ 49. Georges Poull, op. cit., p. 39-42.

${ }^{10}$ Georges POULL, op. cit., p. 43-58.

${ }^{11}$ Emond du BOULLAY, La vie et trépas des princes de paix, le bon duc Antoine et saige duc François premier de leur nom..., Metz, 1547, n. p.

${ }^{12}$ La transcription du testament est donnée dans Georges PoULL, op. cit., p. 131-135.

${ }^{13}$ Georges Poull, op. cit., p. 59-69.

${ }^{14}$ La figure 2 suit en parallèle la dévolution du cartulaire et de l'armorial.
} 
cette tour était fermée par une «porte blindée » dont la serrure est datée de $1537^{15}$. Cette pièce est connue dans la tradition orale comme la salle des archives. Il y a tout lieu de croire que c'est bien dans cette pièce qu'étaient conservées les archives de Nicolas de Lutzelbourg. De toute évidence, le cartulaire n'en est sorti que dans les dernières années du XVIII ${ }^{\mathrm{e}}$ siècle ou dans les premières années du $\mathrm{XIX}^{\mathrm{e}}$ siècle lorsqu'il fut décidé de vendre le château. Le cartulaire rejoignit alors les archives des ducs de Mouchy à Mouchy-le-Châtel ${ }^{16}$. Ces archives furent temporairement déposées dans les années 1980 aux Archives départementales de l'Essonne. Durant cette période, le fonds fut microfilmé et un exemplaire du microfilm du cartulaire fut acquis par les Archives départementales de Meurthe-et-Moselle ${ }^{17}$. Actuellement, la localisation précise du document est inconnue ${ }^{18}$. Il n'est donc pas possible d'en faire une description codicologique.

\section{Le cartulaire :}

On peut cependant dire qu'il se compose d'au moins 143 feuillets. Mais il en compte sans doute davantage puisque les feuillets vierges n'ont pas été photographiés ${ }^{19}$. Aucune indication d'échelle ne permet d'en définir les dimensions.

Seule l'étude du contenu est envisageable. Un texte introductif en donne une définition précise. Ce texte mérite d'être ici intégralement transcris :

\section{Pourquoi et par qui ?}

«En ce livre sont faictes et escriptes les vraies copies de toutes et chacunes les lettres, tiltres et munimens concernans et faisans mencion de toutes les maisons, chasteaulx, terres, seigneuries, rentes, censes, revenues, droictures, heritaiges, possessions, assignations et gaigieres qui de present sont tenues, possedees et qui competent et appartiennent à honnoré seigneur messire Nicolas de Luxembourg, chevalier, seigneur de Fleville, etc. on duché de Bar meismement on duché de Lorraine et conté de Vaudemont dependans et suyvans à son chastel et maison fort de Germeney. Icelles coppies prinses, extraites et collationnees à la requeste dudit seigneur de Fleville aux vraiz originalz par nous, Jehan Picart, tabellion et garde du seel du tabellionnaige de Chastenoy et de Neufchastel, et Françoys de Relenges, aussi tabellion demeurant audit Neufchastel, pour et adfin de mieulx conserver et garder lesdites lettres, tiltres et munymens et obvier à la demolucion des seelz, signatures, parchemin et escriptures d'iceulx que par trop souvent les tenir et manyer se pourroient deperir, degaster et dyminuer meismes aussi pour plus promptement en avoir la veue et lecture quant besoing sera. Iceulx extraictz, copies et colations faictes en l'an mil cinq cens quarante. »

Il s'agit donc d'un livre dans lequel sont copiés tous les actes des biens appartenant à Nicolas de Lutzelbourg, chevalier, seigneur de Fléville qui dépendent de son château de Germiny assis tant dans les duchés de Bar et de Lorraine, qu'au comté de Vaudémont. Ce travail de copie des originaux fut entrepris en 1540 par Jean Picart, tabellion et garde du scel

\footnotetext{
${ }^{15}$ Ibid., p. 66 ; Cécile BERTRAND, op. cit., p. 29.

${ }^{16}$ Oise, ar. Beauvais, c. Noailles.

17 Arch. dép. de Meurthe-et-Moselle, 1 Mi 809. Ce microfilm a été numérisé en 2010 (FRAD054_2NUM_01Mi-00809_E_1).

${ }^{18}$ Le cartulaire était conservé au château de Mouchy dans les années 1980 ; il n'y était vraisemblablement plus en 2010. A cette date, il était peut-être dans la bibliothèque parisienne du duc de Mouchy, décédé en 2011. Le fonds de cette bibliothèque a été rapatrié au château de Mouchy ; l'inventaire est en cours.

${ }^{19} \mathrm{La}$ foliotation ancienne, en chiffres romains, permet de s'en rendre compte ; on passe, par exemple, du feuillet XXXI au feuillet XXXIII.
} 
du tabellionnage de Châtenoy et Neufchâteau, demeurant à Neufchâteau, et de François de Relanges, tabellion, demeurant lui-aussi à Neufchâteau.

Deux objectifs étaient visés : la conservation et la facilité de consultation des documents.

Nicolas eut recours à deux tabellions de Neufchâteau ${ }^{20}$. François de Relanges est inconnu, on peut juste supposer qu'il est originaire du village de Relanges ${ }^{21}$. Jean Picart est en revanche mieux documenté. Originaire de Neufchâteau, il commença sa carrière en 1525 comme tabellion au bailliage de Vôge, dont le siège était à Mirecourt ${ }^{22}$. Il fut nommé conseiller et secrétaire du duc en $1542^{23}$. Enfin, quand en 1546 Nicolas de Lutzelbourg en fait l'un de ses exécuteurs testamentaires, il le qualifie de «lieutenant de bailly et receveur du Neufchastel $»^{24}$.

\section{Contenu :}

Ce cartulaire commence par une longue table composée d'au moins 25 feuillets (non foliotés). Elle se subdivise en différentes parties «[mises] par ordre suyvant le $A B C$ » et commence par les documents concernant Germiny, contenus dans la seizième layette, cotée $\mathrm{Q}$, du «cabinet » ou «tresor» de Nicolas de Lutzelbourg. Viennent ensuite, les chartes concernant Viterne et Crépey ${ }^{25}\left(17^{\mathrm{e}}\right.$ layette, cotée R), Vaudémont ${ }^{26}$ (17 $7^{\mathrm{e}}$ layette, cotée R), Mazirot (18 layette, cotée $S)$, Avillers ${ }^{27}$ (18e layette, cotée $\left.S\right)$, Offroicourt (19 ${ }^{\mathrm{e}}$ layette, cotée $\mathrm{T})$, Haréville ${ }^{28}\left(19^{\mathrm{e}}\right.$ layette, cotée $\left.\mathrm{T}\right)$, Sommerécourt ${ }^{29}\left(20^{\mathrm{e}}\right.$ layette, cotée $\left.\mathrm{V}\right)$, enfin la dernière contient les reprises et dénombrements $\left(21^{\mathrm{e}}\right.$ layette, cotée $\left.\mathrm{X}\right)$. Chaque entrée de cette table contient une brève analyse de l'acte, sa sous-cote et le numéro du feuillet (en chiffre romain) auquel se trouve la transcription. Les sous-cotes, comme les cotes, sont alphabétiques (de A à ŹŹŹ́) mais les actes sont rangés au sein de chaque layette par ordre chronologique. Il est à noter que les pièces originales étaient cotée sur le dos.

La suite du texte donne la transcription des 80 pièces d'archives du chartrier de la seigneurie de Germiny et de ses dépendances tel qu'il était constitué en 1540. Chaque transcription paraît être intégrale, paraît seulement puisque aucun original n'a pu être retrouvé, c'est cependant ce que semble indiquer la comparaison avec trois textes transcrits dans les enregistrements des lettres patentes de René II et d'Antoine ${ }^{30}$. A l'exception de ces trois textes, le cartulaire est actuellement le seul document à conserver le souvenir des actes du chartrier de la seigneurie de Germiny. Les transcriptions sont validées par le seing manuel des deux tabellions : «Picart » et « de Relenges ».

Cinq actes ont été insérés après la réalisation de ce premier ensemble homogène, tant dans la table que dans le corpus de transcriptions. Dans la table, les analyses de ces ajouts sont

\footnotetext{
${ }^{20}$ Vosges, ch.-1. ar. Ceci n'est pas une nouveauté en Lorraine, le cartulaire de Sorbey (Meuse, ar. Verdun, c. Spincourt) est déjà l'œuvre de tabellions dans le premier quart du XVI ${ }^{\mathrm{e}}$ siècle (Lucie FosSIER et Olivier GuYOTJEANNIN, op. cit., p. 385 et 390).

${ }^{21}$ Vosges, ar. Epinal, c. Darney.

${ }^{22}$ Vosges, ar. Neufchâteau, ch.-1. c. ; Arch. dép. de Meurthe-et-Moselle, B 16, fol. 140 (le 28 avril).

${ }^{23}$ AD54, B 22, f. 113 (le 13 août).

${ }^{24}$ Georges PoULL, op. cit., p. 135.

${ }^{25}$ Meurthe-et-Moselle, ar. Nancy, c. Vézelise et ar. Toul, c. Colombey-les-Belles.

${ }^{26}$ Meurthe-et-Moselle, ar. Nancy, c. Vézelise.

${ }^{27}$ Vosges, ar. Epinal, c. Charmes.

${ }^{28}$ Vosges, ar. Neufchâteau, c. Vittel.

${ }^{29}$ Haute-Marne, ar. Chaumont, c. Bourmont.

${ }^{30}$ Textes n 13, 82 et 51 / Arch. dép. de Meurthe-et-Moselle, B 7, fol. 35, B 4, fol. 120 et B 18, fol. 81.
} 
placés à la fin de la partie consacrée à la layette Viterne et Crépey et après la suite et fin des analyses de celle-ci («encores lesdits Viterne et Crepey»). Les transcriptions de ces documents se trouvent aux feuillets : LII $\mathrm{r}^{\circ}$-LIII $\mathrm{r}^{\circ}$ (21 juin 1541, seing manuel Picart), LIII $\mathrm{v}^{\circ}$ (12 juillet 1541, Picart), LIX $\mathrm{r}^{\circ}-\mathrm{LX} \mathrm{v}^{\circ}$ (11 octobre 1540, Picart), feuillet I entre LX et LXIFeuillet II entre LX et LXI (7 novembre 1542, Picart), LXII bis $r^{\circ}-2$ fol. non foliotés ( 20 juin 1541 pas de seing manuel mais même graphie). Ces actes ont donc été transcrits par Jean Picart entre le 11 octobre 1540 et le 7 novembre 1542.

Le classement des chartes est donc essentiellement géographique (Fig. 3 et 4); la layette reprises et dénombrements est une exception. Sur les quatre-vingt cinq actes du cartulaire, cinquante deux $(61,2 \%)$ concernent directement ou indirectement Germiny ${ }^{31}$. Neuf documents $(10,5 \%)$ se rapportent à la terre de Mazirot et à Avillers qui en dépend ; dix-sept pièces $(20 \%)$ touchent la seigneurie d'Offroicourt dont fait partie Haréville et enfin huit chartes $(8,2 \%)$ sont en lien avec la seigneurie dite des Wisse à Sommerécourt qui appartenait en partie aux seigneurs de Germiny. A l'intérieur des layettes le classement est chronologique; les actes les plus anciens sont les premiers. Globalement la répartition chronologique est la suivante : trois actes $(3,5 \%)$ pour le XIII ${ }^{\mathrm{e}}$ siècle $(2$ de 1281 et 1 de $1297)$, vingt-sept $(31,6 \%)$ pour le $\mathrm{XIV}^{\mathrm{e}}$ siècle, vingt-six $(30,5 \%)$ pour le $\mathrm{XV}^{\mathrm{e}}$ siècle et vingtneuf $(34 \%)$ jusqu'en 1542.

Du point de vue typologique les actes se répartissent comme suit :

Reprise de fief : 11

Dénombrement : 1

Donation : 5

Assignation : 1

Vente : 9

Confirmation de vente : 3

Rachat : 1

Engagement et rachat d'engagement : 6

Echange : 1

Accensement : 4

Reconnaissance de dette : 1

Quittance : 4

Traité de mariage : 3

Testament : 1

Accord : 11

Ratification : 3

Autorisation : 1

Mandement : 2

Attestation : 3

Il s'agit essentiellement bien sûr d'actes liés à la seigneurie et à la gestion des biens.

Sept actes ont pour auteur juridique des ecclésiastiques ou des communautés ecclésiastiques (abbesse de Remiremont, abbés de Clairlieu, de Saint-Epvre de Toul, prévôt et chapitre de la collégiale Saint-Jean de Vaudémont). Dans quatre cas, l'auteur de l'acte écrit est le même que celui de l'acte juridique. Les trois autres actes sont des actes où les

\footnotetext{
${ }^{31}$ Il s'agit des actes contenus dans les layettes Q (Germiny), R (Viterne, Crépey, Vaudémont) et X (reprises et dénombrement). Cette dernière layette concerne en fait Germiny.
} 
établissements ecclésiastiques (Remiremont et Saint-Epvre de Toul) sont co-auteurs de l'acte juridique (l'autre auteur est Nicolas de Lutzelbourg); ils sont alors rédigés dans les tabellionnages de Mirecourt et de Châtenois et Neufchâteau. Dix-sept actes émanent de princes ou de leur lieutenant, ducs de Lorraine (Ferry III, Raoul, Charles II, Nicolas, marquis du Pont, lieutenant de Jean d'Anjou, René II et Antoine), ducs de Bar (Henri de Bar, seigneur de Pierrefort, lieutenant de Robert, Robert, René $\mathrm{I}^{\text {er }}$ d'Anjou), comtes de Vaudémont (Henri de Joinville et Antoine de Lorraine) ; dans ces cas, auteurs de l'acte juridique et de l'acte écrit se confondent. L'essentiel du corpus est constitué par quarante-sept actes donnés par des nobles. Cinq sont écrits par les auteurs juridiques eux-mêmes, les quarante-deux autres ont été produits par les officialité de Besançon (1) et de Toul (11), les tabellionnages de Mirecourt (9), de Nancy (10), de Châtenois et Neufchâteau (9), de Vaudémont (1) et enfin par le prévôt de Foug (1). Deux actes concernent des non-nobles, ils ont été passés sous le sceau de l'officialité de Toul. Une vente conclue entre un noble et un non-noble le fut également. Un dernier document émane de roturiers, il a été vraisemblablement rédigé par les tabellions de Châtenois et Neufchâteau. Le cartulaire renferme douze départs de cour, ils ont été données par les Assises du bailliage de Nancy (5) ou de Mirecourt (5) et écrits dans les tabellionnages de ces localités, ou par les Assises du Change de Nancy (2), dans ce cas les écrits ont été produits par l'échevinage de Nancy ou par le lieutenant du bailli. Enfin, deux actes proviennent de la Chambre des Comptes de Bar.

\section{Intérêt et originalité :}

Ce cartulaire est bien sûr un document précieux pour l'étude de la seigneurie. Il permet en effet de comprendre la transmission et la gestion du patrimoine. Il renseigne également sur les comportements de la noblesse lorraine entre la fin $\mathrm{du} \mathrm{XIII}{ }^{\mathrm{e}}$ siècle et la première moitié du $\mathrm{XVI}^{\mathrm{e}}$ siècle, y compris dans son rapport avec les archives. Les pratiques d'archivage d'un seigneur laïc dans les années 1530-1540 sont ici perceptibles. Il existait au château de Fléville un lieu de conservation du chartrier : le « cabinet » ou «tresor ». Ce dernier était situé au rezde-chaussée de l'ancienne tour carrée, qui échut à Claude de Beauvau. Le chartrier était classé par seigneuries et rangé dans des layettes dotées de cotes alphabétiques. Le cartulaire de Germiny contenant la copie du contenu des layettes cotées de $\mathrm{R}$ à $\mathrm{X}$, on peut supposer que de $\mathrm{A}$ à $\mathrm{P}$, les layettes du chartrier concernaient la seigneurie de Fléville et ses dépendances. Un cartulaire consacré à Fléville est attesté dans un recueil généalogique concernant des familles nobles lorraines, composé par un chanoine de Verdun dans les années 1560-1580 ${ }^{32}$.

Composé dans un souci de conservation des documents originaux et d'optimisation des consultations, le cartulaire vise à améliorer la gestion du domaine; il en va de sa sauvegarde et de son augmentation. Ce document n'est ni précurseur, ni isolé en Lorraine. Il existe en effet des cartulaires de seigneurie dès le $\mathrm{XIV}^{\mathrm{e}}$ siècle comme celui d'Apremont ${ }^{33}$. Il n'est pas non plus le premier cartulaire dont la rédaction a été confiée à un ou plusieurs notaires ou tabellions. Dans le premier quart du XVI ${ }^{\mathrm{e}}$ siècle, Warry de Laval ou de La Vaulx, écuyer, demeurant à Marville, fit réaliser un cartulaire concernant « la seigneurye de Sorbey et appartenances d'icelle et le gaingnaige de la Movillie », par un tabellion qui signait «Henriquet ». Jacob de Champbaudour, un autre tabellion, le complèta après $1515^{34}$. Ces cartulaires de petites seigneuries, établis par des notaires, sont courants dans cette période. La troisième et dernière des grandes périodes de rédaction des cartulaires définies par Lucie

\footnotetext{
${ }^{32}$ Une copie de ce recueil, conservé dans une collection privée, a été faite par Georges Poull. Un micro-film en a été réalisé pour les Archives départementales de Meurthe-et-Moselle (Georges PouLL, op. cit., p. 21).

${ }^{33}$ Arch. dép. de Meurthe-et-Moselle, B 508.

${ }^{34}$ Ce cartulaire, conservé dans une collection privée, est connu par un microfilm conservé aux Archives départementales de Meurthe-et-Moselle sous la cote 1 Mi 448 (cf. infra note 20).
} 
Fossier et Olivier Guyotjeannin, s'étend des «décennies médianes du XIV ${ }^{\mathrm{e}}$ siècle jusqu'aux premières décennies du $\mathrm{XVI}^{\mathrm{e}}$ siècle ». Dans le cas de la Lorraine, cette troisième période doit sans doute être étendue au troisième quart du XVI ${ }^{\mathrm{e}}$ siècle. Le cartulaire de la terre de Chambley réalisé dans les années 1560-1570 par un tabellion nommé Aulbertin est, en effet, très proche de par sa conception des cartulaires de Sorbey et de Germiny ${ }^{35}$. La terre de Chambley était alors aux mains de Perrin II de Haraucourt, fils de Jacques de Haraucourt et d'Eve de Bessey, dont le frère Jean avait épousé en 1554 Louise de Lutzelbourg, fille de Nicolas.

\section{Conclusion :}

Le cartulaire de Germiny réalisé pour Nicolas de Lutzelbourg en 1540 ne permet pas de nourrir la réflexion sur les rapports entre les originaux et les cartulaires. Il n'est pas non plus un document extraordinaire puisqu'il fait parti d'un ensemble plus vaste. Mais il convient de lui donner sa place dans l'inventaire des cartulaires médiévaux et modernes qu'entreprend de rassembler la section diplomatique de l'IRHT ${ }^{36}$. Il permet en effet, grâce à l'apport d'une nouvelle documentation, d'enrichir nos connaissances tant sur la seigneurie de Germiny et ses dépendances que sur certains pans encore méconnus de la biographie de Nicolas de Lutzelbourg. A ce titre, l'édition des 85 actes qu'il contient, dont la plupart des originaux paraissent perdus, doit être entreprise. Son intérêt tient aussi au fait qu'il révèle les méthodes et les buts de l'archivage d'un fonds seigneurial : souci de conservation, souci de classement et souci de rassemblement cohérent afin d'assurer la meilleure gestion possible du domaine et sa défense en cas de conflit juridique. S'insérant dans un ensemble plus vaste, il est une pièce qui éclaire la genèse de ces cartulaires de seigneurie dans la Lorraine du $\mathrm{XVI}^{\mathrm{e}}$ siècle. Une confrontation du cartulaire de Germiny avec ceux de Sorbey et de Chambley, et plus généralement avec l'ensemble des cartulaires de seigneurie repérés, devrait être fructueuse.

\footnotetext{
35 Arch. dép. de Meurthe-et-Moselle, E 238. Maxime GrdjAn, Le cartulaire de Chambley, Mémoire de Master 2, Université Nancy2, 2009.

${ }^{36} \mathrm{http}: / / \mathrm{www} . \mathrm{cn}-\mathrm{telma} . \mathrm{fr} / \mathrm{cartulR} / \mathrm{index} /$
} 
ANNEXE 


\section{Cartulaire de Germiny réalisé en 1540 pour Nicolas de Lutzelbourg par Jean Picart, tabellion et garde du sceau du tabellionnage de Châtenois et Neufchâteau, et François de Relanges, tabellion, demeurant à Neufchâteau.}

\begin{tabular}{|c|c|c|c|}
\hline \multicolumn{4}{|c|}{$\begin{array}{l}\text { Contenu de la } 16^{\mathrm{e}} \text { layette cotée } Q \text { du cabinet de Nicolas de Lutzelbourg qui concerne le château de } \\
\text { Germiny (les pièces originales sont « cothee[s] sur le doz») : }\end{array}$} \\
\hline $\mathbf{N}^{\circ}$ & Cote & Analyse & Foliotation \\
\hline 1 & A & $\begin{array}{l}\text { L'official de Toul fait savoir que Vauthier, fils de Vauthier de Toul, } \\
\text { chevalier, a repris en fief de Jean de Germiny, chevalier, tout ce qu'il tient } \\
\text { avec Husson, son frère, à Souveraincourt (1297, } 28 \text { décembre). }\end{array}$ & fol. I $\mathrm{r}^{\mathrm{c}}$ \\
\hline 2 & B & $\begin{array}{l}\text { L'official de Toul fait savoir que Husson, clerc, et Vauthier, écuyer, fils de } \\
\text { feu Vauthier de Toul, chevalier, ont vendu à Jean de Germiny ce qu'ils ont } \\
\text { de par Alix, leur mère, au ban de Souveraincourt pour } 30 \text { livres de toulois } \\
\text { (1303, } 3 \text { juillet). }\end{array}$ & fol. I vo-I \\
\hline 3 & C & $\begin{array}{l}\text { Erard, abbé de Clairlieu, fait savoir qu'il a accensé à Ferry de Germiny et } \\
\text { Alix, sa femme, le moulin et le four de Germiny pour } 4 \text { livres petits tournois } \\
\text { de cens annuel ( } 1337,2 \text { juillet }) \text {. }\end{array}$ & fol. II vo-IIl \\
\hline 4 & D & $\begin{array}{l}\text { L'official de Toul fait savoir qu'Erard du Châtelet, chevalier, et Perrin, son } \\
\text { fils, d'une part, et Ferry de Germiny, écuyer, et Jean, son fils, d'autre part, se } \\
\text { sont accordés au sujet du mariage de Jeanne, fille d' Erard du Châtelet, avec } \\
\text { Jean de Germiny (1345, } 20 \text { août). }\end{array}$ & fol. III v $v^{\circ}$-IIII v \\
\hline 5 & $\mathbf{E}$ & $\begin{array}{l}\text { Le prévôt de Foug fait savoir qu'Adeline de Germiny, fille de feu Jean de } \\
\text { Germiny, chevalier, et veuve d'Henri du Châtelet, seigneur d'Antigny, } \\
\text { chevalier, a reconnu qu'elle a reçu de son père, pour cause de son mariage, la } \\
\text { ville de Mexet entre Haussonville et Saint-Mard, ainsi que } 500 \text { livres de } \\
\text { petits tournois; elle en donne quittance à Ferry de Germiny, écuyer, son } \\
\text { frère, et renonce aux héritages paternels et maternels au profit de ce dernier } \\
(1345,24 \text { avril). }\end{array}$ & fol. $\mathrm{V} \mathrm{r}^{\circ}-\mathrm{V}^{\mathrm{c}}$ \\
\hline 6 & $\mathbf{F}$ & $\begin{array}{l}\text { Le tabellion de Mirecourt fait savoir que Jean du Châtelet, écuyer, a reconnu } \\
\text { qu'il doit à Alix et Isabelle, filles de feu Jean de Germiny, écuyer, et de feue } \\
\text { Jeannette du Châtelet, sa sœur, } 20 \text { livres en vertu du traité de mariage de } \\
\text { Jean de Germiny et de Jeannette (1356, } 11 \text { juillet). }\end{array}$ & fol. VI r \\
\hline 7 & G & $\begin{array}{l}\text { Henri de Bar, seigneur de Pierrefort, lieutenant de Robert, duc de Bar, fait } \\
\text { savoir que comme la maison forte de Germiny a été détruite par Yolande de } \\
\text { Flandre pendant sa régence du duché, il s'est accordé avec Ferry de Germiny } \\
\text { et Oudet et Henri, ses fils, à propos de leur dédommagement (1355, } 9 \\
\text { novembre). } \\
\text { Robert, duc de Bar, marquis du Pont, fait savoir qu'il ratifie l'accord } \\
\text { précédent (1362,12 juillet) }\end{array}$ & $\begin{array}{l}\text { fol. VI v }{ }^{\circ} \text {-VIII r } \\
\text { fol. VIII v }\end{array}$ \\
\hline 9 & $\mathbf{H}$ & $\begin{array}{l}\text { Jean de Raville, chevalier, seigneur de Septfontaines, et Bernard de } \\
\text { Germiny, écuyer, font savoir qu'ils ont conclu le traité de mariage entre } \\
\text { Bernard et Ermenque, fille de Jean de Raville (1451, } 18 \text { décembre). }\end{array}$ & fol. IX $\mathrm{r}^{\circ}-\mathrm{v}^{\mathrm{C}}$ \\
\hline 10 & $\mathbf{J}$ & $\begin{array}{l}\text { L'official de Toul fait savoir que Jacques de Haraucourt, chevalier, seigneur } \\
\text { de Germiny en partie, d'une part, et Bernard de Germiny, écuyer, seigneur } \\
\text { de Germiny en partie, d'autre part, se sont accordés à propos des corvées que } \\
\text { les hommes de Bernard de Germiny doivent à Germiny au lieu-dit le Grand } \\
\text { Ban (1459 (n. s.), } 26 \text { janvier). }\end{array}$ & fol. X r ${ }^{\circ}-X I I I v^{c}$ \\
\hline 11 & $\mathbf{K}$ & $\begin{array}{l}\text { L'official de Toul fait savoir que Jean Tueboix, demeurant à Germiny, et } \\
\text { Clémence, sa femme, ont vendu à Jacques de Germiny et à Eve, sa femme, } \\
\text { une pièce de terre et un pré à Germiny, pour } 10 \text { francs monnaie de Lorraine } \\
(1492,15 \text { décembre). }\end{array}$ & fol. XIIII r ${ }^{\circ}-X V r^{\circ}$ \\
\hline 12 & $\mathbf{L}$ & $\begin{array}{l}\text { Sachent tous que Jacques de Germiny, fils de Bernard de Germiny et } \\
\text { d'Armequine de Raville, et Henri de Raville, fils de Gaspard de Raville et } \\
\text { frère d'Armequine, se sont accordés à propos du différend qui les opposait à } \\
\text { propos du mariage de Bernard de Germiny et d'Armequine de Raville. } \\
\text { L'acte est scellé du sceau du tabellionnage de Nancy ( } 1495,12 \text { juin). }\end{array}$ & fol. XV v $\mathrm{v}^{\circ} \mathrm{XVIII} \mathrm{v}^{\mathrm{c}}$ \\
\hline 13 & $\mathbf{M}$ & $\begin{array}{l}\text { René, roi de Jérusalem et de Sicile, duc de Lorraine et de Bar, fait savoir } \\
\text { qu'il autorise Jacques de Germiny à punir d'une amende de } 70 \text { sous tournois } \\
\text { toutes personnes coupant ou abîmant ses bois de Germiny et d'ailleurs }(1500\end{array}$ & \\
\hline
\end{tabular}




\begin{tabular}{|c|c|c|c|}
\hline & & (n. s.), 17 mars) (voir AD54, B 7, fol. 35). & fol. XIX r r $-v^{\circ}$ \\
\hline 14 & $\mathbf{N}$ & $\begin{array}{l}\text { Sachent tous que Andolffe de la Roche, seigneur de Chastel, a vendu à } \\
\text { Warry de Lutzelbourg, seigneur de Fléville, une moitié de pré sis au ban et } \\
\text { finage de Saint-Dizier à Nancy pour } 160 \text { francs monnaie de Lorraine. L'acte } \\
\text { est scellé du sceau du tabellionnage de Nancy (1509 (n. s.), } 16 \text { février). } \\
\text { Sachent tous que Jacques de Germiny et Eve de la Roche, sa femme, ont } \\
\text { racheté à Warry de Lutzelbourg, seigneur de Fléville, la même moitié de pré } \\
\text { contre } 160 \text { francs monnaie de Lorraine. L'acte est scellé du sceau du } \\
\text { tabellionnage de Nancy (1510,24 avril). }\end{array}$ & fol. $\mathrm{XX} \mathrm{r}^{\circ}-\mathrm{v}^{\circ}$ \\
\hline 16 & $\mathbf{O}$ & $\begin{array}{l}\text { L'official de Toul fait savoir que Martin Flametay, de Germiny, et Catherine, } \\
\text { sa femme, ont vendu à Jacques de Germiny, chevalier, et Eve de la Roche, sa } \\
\text { femme, une vigne à Germiny pour } 15 \text { francs monnaie de Lorraine (1516 (n. } \\
\text { s.), } 25 \text { janvier). }\end{array}$ & fol. XXII r ${ }^{\circ}-v^{\circ}$ \\
\hline 17 & $\mathbf{P}$ & $\begin{array}{l}\text { Sachent tous que Adam Bayer de Boppart, seigneur de Château-Bréhain, et } \\
\text { Jean Bayer de Boppart, seigneur de «Laulnoy », son fils, ont mis en gage à } \\
\text { Jacques de Germiny, chevalier, seigneur dudit lieu, et à Eve de La Roche, sa } \\
\text { femme, } 93 \text { francs } 4 \text { gros monnaie de Lorraine de cens annuel sur la cité de } \\
\text { Toul, ainsi que les grosses et menues dîmes de Vitry et Goviller contre } 2250 \\
\text { francs monnaie de Lorraine. L'acte est scellé du sceau du tabellionnage de } \\
\text { Nancy (1520,13 juin). }\end{array}$ & $\begin{array}{l}\text { fol. XXIII r }{ }^{\circ}- \\
\text { XXIIII r }^{\circ}\end{array}$ \\
\hline 18 & $\mathbf{Q}$ & $\begin{array}{l}\text { L'official de Toul fait savoir que Jacques de Germiny, capitaine de } \\
\text { Neufchâteau, et Eve, sa femme, d'une part, et Humbert Denys, de Germiny, } \\
\text { et Meline, sa femme, d'autre part, ont échangé une pièce de vigne contre un } \\
\text { jardin et } 30 \text { francs monnaie de Lorraine ( } 1522 \text { (n. s.), } 18 \text { février). }\end{array}$ & $\begin{array}{r}\text { fol. XXIIII vo- } \\
\text { XXV r }\end{array}$ \\
\hline 19 & $\mathbf{R}$ & $\begin{array}{l}\text { Sachent tous que Jean de Savigny, seigneur de Leymont, au nom de Claude } \\
\text { de Lutzelbourg, sa femme, d'une part, et Nicolas de Lutzelbourg, chevalier, } \\
\text { seigneur de Fléville, frère de Claude, d'autre part, se sont accordés à propos } \\
\text { de la succession de Jacques de Germiny, chevalier, seigneur dudit lieu, oncle } \\
\text { de Claude et de Nicolas de Lutzelbourg. L'acte est scellé du sceau du } \\
\text { tabellionnage de Nancy (1529,12 octobre). }\end{array}$ & $\begin{array}{r}\text { fol. XXV vo- } \\
\text { XXVI v }\end{array}$ \\
\hline 20 & $\mathbf{S}$ & $\begin{array}{l}\text { Sachent tous qu'Eve de La Roche, veuve de Jacques de Germiny, chevalier, } \\
\text { seigneur dudit lieu, capitaine de Neufchâteau, usufruitière de ses biens, } \\
\text { d'une part, et Nicolas de Lutzelbourg, chevalier, seigneur de Fléville, } \\
\text { capitaine d'Epinal, seul héritier dudit Jacques, d'autre part, se sont accordés } \\
\text { à propos du testament de Jacques de Germiny dont copie est donnée (1527, } \\
14 \text { novembre). L'acte est scellé du sceau du tabellionnage de Nancy (1529, } \\
10 \text { novembre). }\end{array}$ & $\begin{array}{r}\text { fol. XXVII vo- } \\
\text { XXX v }{ }^{\circ}\end{array}$ \\
\hline \multicolumn{4}{|c|}{ Contenu de la $17^{\mathrm{e}}$ layette cotée $\mathbf{R}$ (Viterne et Crépey) : } \\
\hline 21 & $\mathbf{T}$ & $\begin{array}{l}\text { Ferry, duc de Lorraine, fait savoir que Joffroy de Méréville, écuyer, a mis en } \\
\text { gage à Jean de Germiny, sénéchal du duc, ce qu'il a à Viterne contre } 140 \\
\text { petits tournois et } 10 \text { livres de « Nançois » (1302, } 21 \text { août). }\end{array}$ & fol. XXXIIII $r^{\circ}-v^{\circ}$ \\
\hline 22 & $\mathbf{V}$ & $\begin{array}{l}\text { Par devant Jean de Pulligny, chevalier, tenant les assises du commandement } \\
\text { du duc, Jean de Germiny, chevalier, a requis son droit quant au fief de } \\
\text { Viterne qui avait été saisi par le duc ; les assises l'ont remis en sa possession. } \\
\text { L'acte est scellé du sceau de la cour de Nancy (1308, } 21 \text { octobre). }\end{array}$ & fol. $X X X V r^{\circ}-v^{\circ}$ \\
\hline 23 & $\mathbf{X}$ & $\begin{array}{l}\text { Raoul, duc de Lorraine et marquis, fait savoir qu'il a donné à Ferry de } \\
\text { Germiny, écuyer, le fief que tenait Wichard de Méréville, chevalier, à } \\
\text { Viterne (1332, 22 juillet). }\end{array}$ & fol. XXXVI r ${ }^{\circ}$ \\
\hline 24 & $\mathbf{Y}$ & $\begin{array}{l}\text { Raoul, duc de Lorraine et marquis, mande à Wichard de Méréville, } \\
\text { chevalier, de reprendre de Ferry de Germiny ce qu'il tient en fief à Viterne } \\
(1332,31 \text { juillet). }\end{array}$ & fol. XXXVI v ${ }^{\circ}$ \\
\hline 25 & $\mathbf{Z}$ & $\begin{array}{l}\text { Sachent tous que par devant Erard de Haraucourt, bailli de Nancy, tenant les } \\
\text { assises de Nancy, comparut en jugement Jacques de Germiny, écuyer, acteur, } \\
\text { d'une part, contre les forestier, garde des bois et habitants de Viterne, } \\
\text { défendeurs, d'autre part, à propos de certains droits sur les bois de Viterne; } \\
\text { après audition des parties, il est jugé que les défendeurs n'ont pas de droits } \\
\text { sur l'affouage et les amendes des bois de Viterne qui appartiennent à Jacques } \\
\text { de Germiny. Ce départ de cour est scellé des sceaux du tabellionnage de } \\
\text { Nancy et d'Erard de Haraucourt (1505 (n. s.), } 24 \text { février). }\end{array}$ & $\begin{array}{r}\text { fol. XXXVII ro- } \\
\text { XL v }^{\circ}\end{array}$ \\
\hline 26 & Ž & Par devant Jean Harman, de Pulligny, lieutenant du bailli de Nancy, et la & \\
\hline
\end{tabular}




\begin{tabular}{|c|c|c|c|}
\hline & & $\begin{array}{l}\text { justice ordinaire de Nancy comparurent Jean Fevault, maire de Viterne pour } \\
\text { les abbés de Saint-Epvre, acteur, d'une part, et Jacques de Germiny, écuyer, } \\
\text { seigneur de Germiny en partie, capitaine de Neufchâteau, défendeur, d'autre } \\
\text { part, à propos de certaines amendes à percevoir sur Viterne ; après audition } \\
\text { des parties, il est jugé que Jean Fevault n'a pas de droit sur ces amendes. Ce } \\
\text { départ de cour est scellé du sceau de l'échevinage de Nancy ( } 1514,1^{\text {re }} \text { juin). }\end{array}$ & fol. XLI r ${ }^{\circ}$-XLIII r ${ }^{\circ}$ \\
\hline 27 & $\dot{\mathbf{Z}}$ & $\begin{array}{l}\text { Sachent tous que par devant Henri de Lucy, commis à l'office de bailli de } \\
\text { Nancy, tenant les assises de Nancy, comparurent en jugement Jacques de } \\
\text { Germiny, chevalier, seigneur de Germiny, capitaine de Neufchâteau, } \\
\text { demandeur, d'une part, et Jacques de Haraucourt, seigneur de Chambley, } \\
\text { défendeur, d'autre part, à propos de ce que ce dernier tient en fief à Viterne; } \\
\text { les parties sont parvenues à un accord. Ce départ de cour est scellé des } \\
\text { sceaux du tabellionnage de Nancy et d'Henry de Lucy (1527 (n. s.), } 25 \\
\text { février). }\end{array}$ & $\begin{aligned} \text { fol. XLIII v }{ }^{\circ} \\
\quad \text { XLV v } v^{\circ}\end{aligned}$ \\
\hline 28 & $\mathbf{A A}$ & $\begin{array}{l}\text { Par devant Louis de Lescut, lieutenant du bailli de Nancy, et la justice de } \\
\text { Nancy comparurent les habitants de Viterne, acteurs, d'une part, et Jacques } \\
\text { de Germiny, chevalier, seigneur de Germiny et de Viterne, défendeur, } \\
\text { d'autre part, à propos des bois de Viterne; les habitants de Viterne sont } \\
\text { condamnés. Ce départ de justice est scellé du sceau de Louis de Lescut } \\
(1528,30 \text { avril). }\end{array}$ & fol. XLVI $r^{\circ}-$ LI $v^{\circ}$ \\
\hline 29 & $\Delta$ & $\begin{array}{l}\text { Sachent tous qu'en la cour du duc de son tabellionnage de Châtenois et de } \\
\text { Neufchâteau, Etienne Hanry, procureur de Claude Penycier, abbé } \\
\text { commendataire de Saint-Epvre-lès-Toul, et Claude Rychquechiert, prieur } \\
\text { claustral de Saint-Epvre-lès-Toul, en son nom et en celui des religieux de } \\
\text { l'abbaye, d'une part, et Nicolas de Lutzelbourg, chevalier, seigneur de } \\
\text { Fléville, de Germiny en partie, en son nom et en celui de Nicolas de } \\
\text { Dompmartin, seigneur de Germiny en partie, d'autre part, se sont accordés à } \\
\text { propos de la seigneurie du «Grant ban » de Crépey. L'acte est scellé du } \\
\text { sceau du tabellionnage de Châtenois et de Neufchâteau (1541,21 juin). }\end{array}$ & fol. LII $r^{\circ}$-LIII $r^{\circ}$ \\
\hline 30 & & $\begin{array}{l}\text { Claude Penicier, abbé commendataire de Saint-Epvre-lès-Toul, et le couvent, } \\
\text { font savoir qu'ils ont ratifié l'accord précédent ( } 1541,12 \text { juillet). }\end{array}$ & fol. LIII v ${ }^{\circ}$ \\
\hline \multicolumn{4}{|c|}{ « Encores lesditz Viterne et Crepey » : } \\
\hline 31 & BB & $\begin{array}{l}\text { Ferry, duc de Lorraine et marquis, fait savoir qu'il a donné à Jean de } \\
\text { Germiny, chevalier, son sénéchal, le fief que Simon de Marcheville, écuyer, } \\
\text { tenait à Crépey et celui que Renard de Méréville, écuyer, tenait à Viterne } \\
\text { (1303 (n. s.), } 12 \text { mars). }\end{array}$ & fol. LV \\
\hline 32 & $\mathbf{C C}$ & $\begin{array}{l}\text { Ferry, duc de Lorraine et marquis, fait savoir que Simon de Marcheville a } \\
\text { reconnu avoir vendu tout ce qu'il possédait à Crépey à Jean de Germiny pour } \\
1000 \text { livres de messin (1304 (n. s.), } 25 \text { mars). }\end{array}$ & fol. $\mathrm{LV} \mathrm{v}^{\circ}$-LVI r ${ }^{\circ}$ \\
\hline 33 & DD & $\begin{array}{l}\text { Hue, abbé de Saint-Epvre de Toul, et le couvent, font savoir qu'ils ont donné } \\
\text { à Hue dit Tripotel, chevalier, de Neufchâteau, } 60 \text { soudées de terre à toulois à } \\
\text { prendre chaque année sur les rentes de l'abbaye à Crépey (1281, } 21 \text { avril). }\end{array}$ & fol. LVI v ${ }^{\circ}$ \\
\hline 34 & $\mathbf{E E}$ & $\begin{array}{l}\text { Ferry, duc de Lorraine et marquis, fait savoir que les abbés et couvent de } \\
\text { Saint-Epvre de Toul ont donné à Hue dit Tripotel, de Neufchâteau, chevalier, } \\
60 \text { soudées de terre à toulois à prendre sur ce qu'ils ont à Crépey avec son } \\
\text { autorisation ( } 1281,28 \text { avril). }\end{array}$ & fol. LVII $r^{\circ}$ \\
\hline 35 & FF & $\begin{array}{l}\text { L'official de Toul fait savoir que Simon de Marcheville, écuyer, fils de Hue } \\
\text { dit Tripotel, chevalier, de Neufchâteau, a reconnu qu'il tient en foi et } \\
\text { hommage de Jean de Germiny, chevalier, la vouerie de Crépey et tout ce } \\
\text { qu'il peut avoir au ban et au finage de Crépey et qu'il les a vendu à Jean de } \\
\text { Germiny contre } 1000 \text { livres de messin (1304 (n. s.), } 31 \text { mars) }\end{array}$ & $\begin{array}{l}\text { fol. LVII vo- } \\
\text { LVIII v }^{\circ}\end{array}$ \\
\hline 36 & 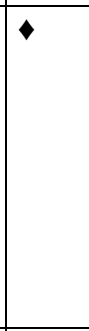 & $\begin{array}{l}\text { Sachent tous que par devant Gérard de Haraucourt, bailli de Nancy, tenant } \\
\text { les assises de Nancy, comparurent en jugement Nicolas de Lutzelbourg, } \\
\text { chevalier, seigneur de Fléville, demandeur, d'une part, contre Poirot, maire } \\
\text { de Crépey, défendeur, d'autre part, à propos de certains droits à lever par le } \\
\text { maire sur les habitants du «Grant ban » de Crépey au profit de Nicolas de } \\
\text { Lutzelbourg; après audition des parties, il est jugé que les droits de ce } \\
\text { dernier sont bons. Ce départ de cour est scellé des sceaux du tabellionnage } \\
\text { de Nancy et de Gérard de Haraucourt (1540,11 octobre). }\end{array}$ & fol. LIX $\mathrm{r}^{\circ}-\mathrm{LX} \mathrm{v}^{\circ}$ \\
\hline 37 & $\Delta$ & Sachent tous qu'en la cour du duc de son tabellionnage de Châtenois et de & \\
\hline
\end{tabular}




\begin{tabular}{|c|c|c|c|}
\hline & & $\begin{array}{l}\text { Neufchâteau, Etienne Henry, prêtre, vicaire de Claude Penicier, abbé } \\
\text { commendataire de Saint-Epvre-lès-Toul, et Nicolas de Lutzelbourg, } \\
\text { chevalier, seigneur de Fléville, de Germiny en partie, en son nom et en celui } \\
\text { de Nicolas de Dompmartin, seigneur de Germiny en partie, et Poirot Maire, } \\
\text { mayeur du «Grant ban » de Crépey, Epvre Thiebault, échevin audit lieu, et } \\
\text { Balthasar Ferrans, demeurant à Crépey, au nom des habitants dudit lieu, } \\
\text { d'autre part, se sont accordés à propos de la vente du bois de Crépey. L'acte } \\
\text { est scellé du sceau du tabellionnage de Châtenois et de Neufchâteau (1542, } 7 \\
\text { novembre). }\end{array}$ & $\begin{array}{l}\text { fol. I entre le LX et } \\
\text { LXI r }{ }^{\circ} \text {-fol. II entre } \\
\text { LX et LXI r }\end{array}$ \\
\hline 38 & & $\begin{array}{l}\text { Mention de la ratification de l'accord précédent par les habitants de Crépey } \\
(1542,19 \text { novembre). }\end{array}$ & $\begin{array}{r}\text { fol. II entre LX et } \\
\text { LXI r }{ }^{\circ}\end{array}$ \\
\hline 39 & & $\begin{array}{l}\text { Sachent tous que par devant Gérard de Haraucourt, bailli de Nancy, tenant } \\
\text { les assises de Nancy, comparurent en jugement Jacob de Haraucourt, } \\
\text { seigneur de Chambley, Nicolas de Lutzelbourg, chevalier, seigneur de } \\
\text { Fléville, et François de Dompmartin, seigneur dudit lieu, seigneurs voués de } \\
\text { Viterne, demandeurs, d'une part, contre le maire de Viterne, défendeur, } \\
\text { d'autre part, à propos de certains droits à Viterne. Un accord amiable est } \\
\text { convenu entre les parties. Ce départ de cour est scellé des sceaux du } \\
\text { tabellionnage de Nancy et de Gérard de Haraucourt (1541,20 juin). }\end{array}$ & $\begin{array}{l}\text { fol. LXII (bis) }{ }^{\circ}- \\
\text { non folioté }\end{array}$ \\
\hline \multicolumn{4}{|c|}{ Contenu de la $17^{\mathrm{e}}$ layette cotée $\mathrm{R}$ (Vaudémont) : } \\
\hline 40 & GG & $\begin{array}{l}\text { Henri, comte de Vaudémont, seigneur de Joinville, fait savoir qu'il a donné à } \\
\text { Oudot de Germiny, écuyer, une grange sise à Vaudémont }(1363,10 \\
\text { septembre). }\end{array}$ & fol. LXII r ${ }^{\circ}$ \\
\hline 41 & HH & $\begin{array}{l}\text { Béatrice de Rambervillers, femme de feu Oudot de Germiny, écuyer, et } \\
\text { Ferry de Germiny, son fils, garant de Oudart le petit, son frère, et de toutes } \\
\text { ses souurs, font savoir qu'ils ont accensé à Jean, fils de Jean Leclerc de } \\
\text { Vaudémont, et à Babbeil, sa femme, } 5 \text { sous de cens annuel sur une maison } \\
\text { sise à Vaudémont contre } 8 \text { francs d'or d'entrée que Béatrice et son fils } \\
\text { reconnaissent avoir reçu ( } 1385,25 \text { juin ?) }\end{array}$ & fol. LXII vo \\
\hline 42 & JJ & $\begin{array}{l}\text { Geoffroy de Fontenoy, écuyer, seigneur de Praye en partie, fait savoir qu'il a } \\
\text { repris en fief à Ferry de Germiny, écuyer, un pré sis au finage de Praye } \\
(1396,10 \text { juillet). }\end{array}$ & fol. LXIII $r^{\circ}$ \\
\hline 43 & KK & $\begin{array}{l}\text { Le prévôt et le chapitre de l'église collégiale Saint-Jean de Vaudémont font } \\
\text { savoir que pour la concession du patronage laïque de l'église de Hardéval } \\
\text { près de Vézelise que leur ont fait Ferry de Germiny et son neveu Jean par } \\
\text { lettres du } 15 \text { octobre } 1418 \text {, ils célèbreront diverses messes pour le salut de } \\
\text { l'âme de Ferry, Jeanne de Mazirot, sa femme et Jean ( } 1418,15 \text { octobre). }\end{array}$ & $\begin{array}{l}\text { fol. LXIII v }{ }^{\circ}- \\
\text { LXIIII } r^{\circ}\end{array}$ \\
\hline 44 & LL & $\begin{array}{l}\text { Sachent tous que Jacques de Germiny, écuyer, seigneur de Germiny en } \\
\text { partie, en son nom et en celui d'Eve de La Roche, sa femme, a accensé à } \\
\text { Nicolas Gerrard, de Saulxures-lès-Bulgnéville, et à Catherine, sa femme, une } \\
\text { maison sise à Vaudémont et différents biens fonciers, contre } 1 \text { florin d'or et } \\
\text { deux chapons payables à la Saint-Martin d'hiver. L'acte est scellé du sceau } \\
\text { du tabellionnage de Vaudémont (1504 (n. s.), } 31 \text { janvier). }\end{array}$ & $\begin{array}{l}\text { fol. LXIIII v }{ }^{\circ}- \\
\text { LXVI } r^{\circ}\end{array}$ \\
\hline \multicolumn{4}{|c|}{ Contenu de la $18^{\mathrm{e}}$ layette cotée $S$ (Mazirot) : } \\
\hline 45 & MM & $\begin{array}{l}\text { Sachent tous qu'en la cour du duc de son tabellionnage de Mirecourt, Perrin } \\
\text { de Mazirot, écuyer, en vertu du traité de son mariage avec Isabelle, fille de } \\
\text { Gérard de Mirecourt, chevalier, a bien reçu les sommes et biens convenus. } \\
\text { L'acte est scellé du sceau du tabellionnage de Mirecourt (1338, } 7 \text { mai). }\end{array}$ & $\begin{array}{l}\text { fol. LXX r }{ }^{\circ} \\
\text { LXXII v }^{\circ}\end{array}$ \\
\hline 46 & NN & $\begin{array}{l}\text { Sachent tous que comme Huguenin de Mazirot et Ferry de Germiny, } \\
\text { écuyers, voués de Haréville en partie, ont quitté les habitants dudit lieu de la } \\
\text { taille qu'on dit «la taille de may », ces derniers s'engagent à repayer cette } \\
\text { taille quand il plaira à Huguenin et Ferry de leur redemander (1404, } 10 \text { août). }\end{array}$ & fol. LXXIII r ${ }^{\circ}-v^{\circ}$ \\
\hline 47 & OO & $\begin{array}{l}\text { Sachent tous que par devant Gérard de Ligniville, écuyer, bailli de Vôge } \\
\text { tenant les assises de Mirecourt, fut jugé le procès opposant Henri de } \\
\text { Marches, écuyer, et Gillette de Mazirot, sa femme, à Waltrin de Germiny, } \\
\text { écuyer, à propos du château de Mazirot. Ce départ de cour est scellé des } \\
\text { sceaux du tabellionnage de Mirecourt (1474, } 2 \text { mai). }\end{array}$ & $\begin{array}{l}\text { fol. LXXIIII } r^{\circ} \\
\text { LXXVI }^{\circ}\end{array}$ \\
\hline 48 & $\mathbf{P P}$ & $\begin{array}{l}\text { Sachent tous que Ferry de Germiny, fils de feu Jean de Germiny, écuyer, } \\
\text { seigneur de Mazirot en partie, a reconnu qu'il a engagé à Béatrix de } \\
\text { Germiny, veuve de Philibert du Châtelet, tout ce qu'il possède au château et }\end{array}$ & \\
\hline
\end{tabular}




\begin{tabular}{|c|c|c|c|}
\hline 50 & & $\begin{array}{l}\text { à la ville de Mazirot, à Tantimont, Xaronval, Bouxurulles et Chauffecourt } \\
\text { contre } 1000 \text { francs monnaie de Lorraine. . L'acte est scellé du sceau du } \\
\text { tabellionnage de Nancy (1478 (n. s.), } 23 \text { février). } \\
\text { Sachent tous qu'Endolphe de Lutzelbourg, chevalier, seigneur de Fléville, } \\
\text { d'une part, et Biétrix de Germiny, veuve de Philibert du Châtelet, fille de feu } \\
\text { Bernard de Germiny, écuyer, seigneur d'Offroicourt en partie, Jacquot et } \\
\text { Ferry, ses frères, d'autre part, se sont accordés à propos du traité de mariage } \\
\text { de Warry de Lutzelbourg, fils d'Endolphe, et de Biétrix. L'acte est scellé du } \\
\text { sceau du tabellionnage de Mirecourt (1482, } 22 \text { août). } \\
\text { Sachent tous que comme Ferry de Germiny, écuyer, fils de feu Jean de } \\
\text { Germiny, a engagé à Béatrix de Germiny, tout ce qu'il possède au château et } \\
\text { à la ville de Mazirot, à Tantimont, Xaronval, Bouxurulles et Chauffecourt } \\
\text { par lettres du } 23 \text { février } 1478 \text {, il renonce à ses possessions et les donne en } \\
\text { vertu du traité de mariage ci-dessus à ladite Béatrix. L'acte est scellé du } \\
\text { sceau du tabellionnage de Nancy (1482, } 24 \text { décembre). }\end{array}$ & $\begin{array}{l}\text { fol. LXXVII r }{ }^{\circ}- \\
\qquad \text { LXXVIII v }{ }^{\circ}\end{array}$ \\
\hline 51 & QQ & $\begin{array}{l}\text { Antoine, duc de Calabre, de Lorraine et de Bar, marquis, marquis du Pont, } \\
\text { comte de Provence, de Vaudémont, etc. fait savoir qu'il a donné à son } \\
\text { conseiller et capitaine d'Epinal, Nicolas de Lutzelbourg, chevalier, seigneur } \\
\text { de Fléville, différentes rentes et revenus à prendre sur les bans d'Avillers, de } \\
\text { Mazirot et de Chauffecourt (1531 (n. s.), } 4 \text { avril) (voir AD54, B 18, fol. 81). }\end{array}$ & $\begin{array}{r}\text { fol. } \text { LXXXIII v }^{\circ}- \\
\text { LXXXIIII v }^{\circ}\end{array}$ \\
\hline \multicolumn{4}{|c|}{ Contenu de la $18^{\mathrm{e}}$ layette cotée $S$ (Avillers) : } \\
\hline 52 & RR & $\begin{array}{l}\text { Sachent tous que Wichard d'Amance, écuyer, et Jeanne, sa femme, ont } \\
\text { donné à Perrin de Mazirot, écuyer, et à Isabelle, sa femme, tout ce qu'ils ont } \\
\text { hérité de feu Jean de Bayon, chevalier, à Avillers. L'acte est scellé du sceau } \\
\text { du tabellionnage de Nancy (1352, } 29 \text { septembre). }\end{array}$ & fol. LXXXVII $\mathrm{r}^{\circ}$ \\
\hline 53 & SS & $\begin{array}{l}\text { Sachent tous que l'abbesse et le couvent de Remiremont, Nicolas de } \\
\text { Lutzelbourg, chevalier, seigneur de Fléville, et Jean de Barbay, seigneur de } \\
\text { Mazirot en partie, ont accensé aux habitants de Bouxurulles trois bois, sis au } \\
\text { ban d'Avillers. L'acte est scellé du sceau du tabellionnage de Mirecourt } \\
\text { (1537 (n. s.), } 14 \text { mars). }\end{array}$ & $\begin{array}{l}\text { fol. LXXXVII v }{ }^{\circ}- \\
\text { LXXXXIII r }^{\circ}\end{array}$ \\
\hline \multicolumn{4}{|c|}{ Contenu de la $19^{\mathrm{e}}$ layette cotée $\mathrm{T}$ (Offroicourt) : } \\
\hline 54 & TT & $\begin{array}{l}\text { Sachent tous que Perrin de Mazirot a repris en fief de Renier d'Offroicourt } \\
\text { ce qu'il tient de lui à Mazirot. . L'acte est scellé du sceau du tabellionnage de } \\
\text { Mirecourt (1363 (n. s.), } 5 \text { janvier). }\end{array}$ & $\begin{array}{l}\text { fol. } \mathrm{LXXXXVII} \\
\qquad \mathrm{r}^{\circ}-\mathrm{v}^{\circ}\end{array}$ \\
\hline 55 & VV & $\begin{array}{l}\text { Sachent tous que Gérard de Mazirot, chevalier, et Huguenin, son frère, } \\
\text { écuyer, se sont accordés à propos de la seigneurie d'Offroicourt, héritée de } \\
\text { Renier d'Offroicourt, chevalier, qu'ils se partagent par moitié. L'acte est } \\
\text { scellé du sceau du tabellionnage de Mirecourt (1378, } 1^{\text {er juin }) \text {. }}\end{array}$ & fol. LXXXXVIII r ${ }^{\circ}$ \\
\hline 56 & $\mathbf{X X}$ & $\begin{array}{l}\text { Jeanne de Mazirot, dame de Germiny, fait son testament. L'acte est scellé du } \\
\text { sceau de l'official de Toul (1439, } 3 \text { avril). }\end{array}$ & $\begin{array}{r}\text { fol. LXXXXVIII r }{ }^{\circ}- \\
\text { LXXXXIX v }^{\circ}\end{array}$ \\
\hline 57 & YY & $\begin{array}{l}\text { Sachent tous que par devant Jacques de Savigny, écuyer, bailli de Vôge } \\
\text { tenant les assises de Mirecourt, fut jugé le procès opposant André de Ville, } \\
\text { seigneur de Domjulien, à Bernard de Germiny, écuyer, à propos d'un cheval } \\
\text { et harnois appartenant à Bernard de Germiny saisis par les officiers d'André } \\
\text { de Ville. Ce départ de cour est scellé du sceau du tabellionnage de Mirecourt } \\
(1461,30 \text { novembre). }\end{array}$ & $r^{0}-v^{\circ}$ \\
\hline 58 & $\mathbf{Z Z}$ & $\begin{array}{l}\text { Sachent tous qu'en la cour du duc de son tabellionnage de Châtenoy et de } \\
\text { Neufchâteau, Jean de Toulon, chevalier, et Yolande de Haussonville, sa } \\
\text { femme, d'une part et Endolffe de Lutzelbourg, d'autre part, ont fait le } \\
\text { partage de la maison forte d'Offroicourt. L'acte est scellé du sceau du } \\
\text { tabellionnage de Châtenoy et de Neufchâteau (1466 (n. s.), } 20 \text { janvier). }\end{array}$ & fol. CI $r^{\circ}$ \\
\hline 59 & 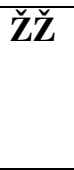 & $\begin{array}{l}\text { Jean de Ligniville, chevalier, seigneur dudit lieu et de Tantonville, fait savoir } \\
\text { qu'il tient en foi et hommage de Jacques de Germiny, écuyer, seigneur } \\
\text { d'Offroicourt, le quart de la seigneurie d'Offroicourt et en donne son } \\
\text { dénombrement (1481 (n. s.), } 10 \text { mars). }\end{array}$ & fol. CIIII r ${ }^{\circ}-v^{\circ}$ \\
\hline 60 & ŹŹ́ & $\begin{array}{l}\text { Sachent tous que par devant Henri de Ligniville, chevalier, bailli de Vôge } \\
\text { tenant les assises de Mirecourt, fut jugé le procès opposant les seigneurs } \\
\text { d'Offroicourt, voués de Ahéville en partie, d'une part, et Pierre Perrin, de } \\
\text { Mirecourt, d'autre part, à propos de revenus en nature que Pierre prend } \\
\text { chaque année à Ahéville. Ce départ de cour est scellé des sceaux du }\end{array}$ & \\
\hline
\end{tabular}




\begin{tabular}{|c|c|c|c|}
\hline & & tabellionnage de Mirecourt (1493, 20 mai). & fol. $\mathrm{CV} \mathrm{r}^{\circ}-\mathrm{CVI} \mathrm{v}^{\circ}$ \\
\hline 61 & AAA & $\begin{array}{l}\text { Sachent tous qu'en la cour du duc de son tabellionnage de Châtenoy et de } \\
\text { Neufchâteau, Humbert de Serrières, écuyer, seigneur de Resaincourt, haut } \\
\text { voué de Nomeny, a vendu à Nicolas de Lutzelbourg, chevalier, seigneur de } \\
\text { Fléville, et à Marguerite de Lucy, sa femme, la moitié du château bas } \\
\text { d'Offroicourt contre } 4200 \text { francs. L'acte est scellé du sceau du tabellionnage } \\
\text { de Châtenoy et de Neufchâteau ( } 1539,11 \text { septembre). }\end{array}$ & fol. CVII r ${ }^{\circ}-\mathrm{CIX}^{\circ}$ \\
\hline \multicolumn{4}{|c|}{ Contenu de la $19^{\mathrm{e}}$ layette cotée T (Haréville) : } \\
\hline 62 & BBB & $\begin{array}{l}\text { L'official de Toul fait savoir qu'Eudes de Charmes, chevalier, a confirmé la } \\
\text { vente faite par Vautrin de Beaumont, fils de feu Jean de Jaillon, écuyer, à } \\
\text { Gérard de Mirecourt, bailli de Vôge, de tout ce qu'il possède à Haréville } \\
\left(1322,1^{\text {er }} \text { mai). }\right.\end{array}$ & fol. CXII r ${ }^{\circ}$ \\
\hline 63 & CCC & Même confirmation de vente $(1322,14$ juin $)$ & \\
\hline 64 & DDD & $\begin{array}{l}\text { Liébaut de Rosières, chevalier, seigneur de Lignéville, fait savoir qu'il } \\
\text { confirme la vente faite par Jean de «Sygneure », écuyer, et Alix, sa femme, } \\
\text { à Gérard de Mirecourt, bailli de Vôge, de tout ce qu'ils ont à Haréville } \\
\text { (1323, } 7 \text { mai). }\end{array}$ & fol. CXIII r ${ }^{\circ}$ \\
\hline 65 & EEE & $\begin{array}{l}\text { Jean de « Saguenoe », écuyer, et Alix, sa femme, mandent aux maire, aux } \\
\text { prud'hommes et à la communauté de Haréville de payer et de répondre } \\
\text { désormais à Gérard de Mirecourt comme ils le faisaient à eux auparavant } \\
(1323,7 \text { mai). }\end{array}$ & fol. CXIII vo \\
\hline 66 & FFF & $\begin{array}{l}\text { L'official de Besançon fait savoir que Jean de «Saguenoie» et Alix, sa } \\
\text { femme, ont vendu à Gérard de Mirecourt, bailli de Vôge, fils de feu Guiat de } \\
\text { Fontenoy, tout ce qu'ils possèdent à Haréville contre } 36 \text { petits tournois de } \\
\text { Metz (1323, } 28 \text { mai). }\end{array}$ & $\begin{array}{r}\text { fol. } \text { CXIIII r }^{\circ}- \\
\text { CXV r }^{\circ}\end{array}$ \\
\hline 67 & GGG & $\begin{array}{l}\text { Sachent tous qu'en la cour du duc de son tabellionnage de Mirecourt, Eudes } \\
\text { de Charmes, chevalier, a quitté Gérard de Mirecourt, chevalier, bailli de } \\
\text { Vôge, de l'hommage qu'il lui devait pour ce qu'il avait acheté à Vautrin de } \\
\text { Belmont à Haréville qui était du fief d'Eudes. L'acte est scellé du sceau du } \\
\text { tabellionnage de Mirecourt (1324, } 20 \text { décembre). }\end{array}$ & fol. $\mathrm{CXV} \mathrm{v}^{\circ}$ \\
\hline 68 & HHH & $\begin{array}{l}\text { Sachent tous que par devant Karlet de Ville, écuyer, bailli de Vôge tenant les } \\
\text { assises de Mirecourt, fut jugé le procès opposant Mougin dit Gray Bouvel de } \\
\text { Remoncourt, prévôt dudit lieu au nom du duc de Lorraine, d'une part, et } \\
\text { Mougin Grant Jehan de Haréville, sujet de Bernard de Germiny, à propos } \\
\text { d'un essaim d'abeilles trouvé par ce dernier sur le ban de Haréville. Ce } \\
\text { départ de cour est scellé du sceau du tabellionnage de Mirecourt (1463 (n. } \\
\text { s.), } 21 \text { février). }\end{array}$ & fol. $\mathrm{CXVI} \mathrm{r} \mathrm{r}^{\circ}$ \\
\hline 69 & JJJ & $\begin{array}{l}\text { Sachent tous que par devant Collignon de Ville, bailli de Vôge, a été jugé le } \\
\text { jugeant le procès opposant Jean Villain, prévôt de Remoncourt, d'une part, à } \\
\text { Thiriet de Haréville et aux seigneurs d'Haréville, d'autre part, à propos } \\
\text { d'amendes que le prévôt demande à Thiriet. Ce départ de cour est scellé du } \\
\text { sceau du tabellionnage de Mirecourt ( } 1472,19 \text { octobre). }\end{array}$ & fol. CXVII r ${ }^{\circ}-v^{\circ}$ \\
\hline 70 & KKK & $\begin{array}{l}\text { Ludwig de Reinach, chevalier, seigneur de Saint-Baslemont, reconnât qu'il } \\
\text { tient en fief de Ferry de Germiny, seigneur d'Offroicourt, ce qu'il a à } \\
\text { Offroicourt (1507, } 24 \text { août). }\end{array}$ & fol. CXVIII ro \\
\hline \multicolumn{4}{|c|}{ Contenu de la $20^{\mathrm{e}}$ layette cotée $\mathrm{V}(«$ Semerecourt $»)$ : } \\
\hline 71 & $\mathbf{L L L}$ & $\begin{array}{l}\text { Sachent tous qu'en la cour du duc de son tabellionnage de Châtenois et } \\
\text { Neufchâteau, Durand de la Nueveville au Boix, demeurant à Sommerécourt, } \\
\text { et seigneur en partie de ce lieu, et Isabelle, sa femme, ont vendu à Jean et } \\
\text { Arnould Wisse, frères et seigneurs en partie de Sommerécourt, différents } \\
\text { biens sis à Sommerécourt contre } 13 \text { francs de bon or (1407, } 24 \text { avril). }\end{array}$ & fol. CXXII r ${ }^{\circ}-v^{\circ}$ \\
\hline 72 & MMM & Autre vente des mêmes (1408 (n. s.), 18 février). & $\begin{array}{r}\text { fol. CXXIII ro- } \\
\text { CXXIIII v }^{\circ}\end{array}$ \\
\hline 73 & NNN & $\begin{array}{l}\text { Sachent tous qu'en la cour du duc de son tabellionnage de Châtenois et de } \\
\text { Neufchâteau, Nicolas du Châtelet, écuyer, seigneur de Vauvillers, tuteur des } \\
\text { enfants de son frère Renaud, d'une part, et Olry Wisse de Gerbévillers, } \\
\text { écuyer, seigneur de Gerbévillers et de Bauzemont, d'autre part, se sont } \\
\text { accordés à propos du rachat de deux engagements jadis faits sur la seigneurie } \\
\text { de Sommerécourt contre } 160 \text { francs que Nicolas a reçu d'Olry. L'acte est } \\
\text { scellé du sceau du tabellionnage de Châtenois et de Neufchâteau (1493, } 12\end{array}$ & fol. CXXV r- \\
\hline
\end{tabular}




\begin{tabular}{|c|c|c|c|}
\hline & & septembre). & CXXVI r $^{\circ}$ \\
\hline 74 & 000 & $\begin{array}{l}\text { Sachent tous qu'en la cour du duc de son tabellionnage de Châtenois et de } \\
\text { Neufchâteau, Olry Wisse de Gerbévillers, seigneur de Gerbévillers et de } \\
\text { Bouzemont, d'une part, et Jacques de Germiny, écuyer, seigneur de Germiny } \\
\text { en partie, d'autre part, se sont accordés à propos du testament de Godefroy } \\
\text { de Bouzemont (1465, } 15 \text { juin) dans lequel ce dernier léguait à feu Bernard de } \\
\text { Germiny, père de Jacques, ainsi qu'à feue Bietrix de Germiny, jadis femme } \\
\text { de Godefroy, souur de Bernard, certains biens sis à Sommerécourt. L'acte est } \\
\text { scellé du sceau du tabellionnage de Châtenois et de Neufchâteau (1501 (n. } \\
\text { s.), } 19 \text { janvier). }\end{array}$ & $\begin{array}{r}\text { fol. } \mathrm{CXXVI} \mathrm{v}^{\circ}- \\
\mathrm{CXXVII} \mathrm{v}^{\circ}\end{array}$ \\
\hline 75 & PPP & $\begin{array}{l}\text { Sachent tous que comme la seigneurie dite des Wisse sise à Sommerécourt } \\
\text { avait jadis été engagée, notamment aux ancêtres d'Antoine et Jacques du } \\
\text { Châtelet, frères, seigneurs du Châtelet, ces derniers consentent au rachat } \\
\text { d'un quart de cette seigneurie par Jacques de Germiny. L'acte est scellé du } \\
\text { sceau du tabellionnage de Nancy }(1512,28 \text { juillet) }\end{array}$ & $\begin{array}{r}\text { fol. CXXVIII r }{ }^{\circ} \\
\text { CXXIX r }^{\circ}\end{array}$ \\
\hline 76 & QQQ & $\begin{array}{l}\text { Sachent tous qu'en la cour du duc de son tabellionnage de Châtenois et de } \\
\text { Neufchâteau, Antoine du Châtelet, écuyer, seigneur du Châtelet et de Sorcy a } \\
\text { reconnu qu'il a consenti au rachat par Jacques de Germiny d'un quart de la } \\
\text { « seigneurie des Wisse » sise à Sommerécourt jadis engagé. L'acte est scellé } \\
\text { du sceau du tabellionnage de Châtenois et de Neufchâteau (1517, } 21 \text { octobre) }\end{array}$ & $\begin{array}{r}\text { fol. } \mathrm{CXXIX} \mathrm{v}^{\circ}- \\
\mathrm{CXXX} \mathrm{r}^{\circ}\end{array}$ \\
\hline 77 & RRR & $\begin{array}{l}\text { Sachent tous que Jacques du Châtelet, chevalier, seigneur de Sorcy, et } \\
\text { Nicolas de Lutzelbourg, chevalier, seigneur de Fléville, capitaine d'Epinal, } \\
\text { se sont accordés à propos du différend qui les opposait à propos de la } \\
\text { seigneurie de Sommerécourt. L'acte est scellé du sceau du tabellionnage de } \\
\text { Mirecourt (1530,18 mars). }\end{array}$ & $\begin{array}{r}\text { fol. } \text { CXXXI r }^{\circ} \\
\text { CXXXII r } \\
\end{array}$ \\
\hline \multicolumn{4}{|c|}{ Contenu de la $21^{\mathrm{e}}$ layette cotée $\mathrm{X}$ (Reprises et dénombrements) : } \\
\hline 78 & SSS & $\begin{array}{l}\text { Charles, duc de Lorraine et marquis, fait savoir que Ferry de Germiny, } \\
\text { écuyer, a repris, en accroissement de ce qu'il tient déjà du duc, la garde de la } \\
\text { cure de Houdelmont (1411 (n. s.), } 15 \text { mars). }\end{array}$ & fol. CXXX \\
\hline 79 & TTT & $\begin{array}{l}\text { René, fils du roi de Jérusalem et de Sicile, duc de Bar, marquis du Pont, } \\
\text { comte de Guise, fait savoir qu'Oudet de Germiny, écuyer, a repris ce qu'il } \\
\text { tient de lui en fief et hommage au bailliage de Bar et ailleurs ; il donne } \\
\text { mandement à son prévôt de Bar de lui en laisser la jouissance à condition } \\
\text { qu'il en fasse le dénombrement dans les quarante jours (Foug, 1430, } 4 \\
\text { novembre). }\end{array}$ & fol. CXXXVI v ${ }^{\circ}$ \\
\hline 80 & VVV & $\begin{array}{l}\text { Antoine de Lorraine, comte de Vaudémont, seigneur de Rumigny, de Boves, } \\
\text { de Joinville, et sénéchal de Champagne, fait savoir qu'il a reçu l'aveu et } \\
\text { dénombrement (dont le texte est donné) de Bernard de Germiny, écuyer, } \\
\text { seigneur d'Offroicourt en partie, pour ce que ce dernier tient de lui à } \\
\text { Vaudémont (Vézelise, } 1451,1^{\text {er }} \text { mars). }\end{array}$ & fol. CXXXVII \\
\hline 81 & XXX & $\begin{array}{l}\text { Nicolas, marquis du Pont, lieutenant de Jean d'Anjou, duc de Lorraine, fait } \\
\text { savoir que Bernard de Germiny a repris ce qu'il tient de lui au duché de } \\
\text { Lorraine et spécialement la garde du curé de Houdelmont et qu'il lui a } \\
\text { enjoint d'en faire le dénombrement dans le délai accoutumé (Nancy, 1465, } \\
22 \text { novembre). }\end{array}$ & fol. CXXXVIII r ${ }^{\circ}$ \\
\hline 82 & Z'Z'Z' & $\begin{array}{l}\text { René, duc de Lorraine et de Bar, etc. fait savoir que Jacques de Germiny, son } \\
\text { conseiller et panetier, a repris ce qu'il tient de lui aux duchés de Lorraine et } \\
\text { de Bar et au comté de Vaudémont et qu'il lui a enjoint d'en faire le } \\
\text { dénombrement dans les quarante jours (Neufchâteau, 1491, } 31 \text { octobre) (voir } \\
\text { AD54, B 4, fol. 120). }\end{array}$ & fol. CXXXVIII v ${ }^{\circ}$ \\
\hline 83 & ZZZ & $\begin{array}{l}\text { Les gens des comptes du duché de Bar font savoir qu'ils ont reçu le } \\
\text { dénombrement de ce que tient Jacques de Germiny à Germiny (1495, } 24 \\
\text { août). }\end{array}$ & fol. CXXXIX r ${ }^{\circ}$ \\
\hline 84 & $\mathbf{Z} \mathbf{Z} \mathbf{Z Z Z}$ & $\begin{array}{l}\text { Antoine, duc de Calabre, de Lorraine et de Bar, etc. aux président et } \\
\text { auditeurs de la chambre des comptes de Bar, et autres ses officiers de } \\
\text { Barrois, fait savoir que Nicolas de Lutzelbourg, chevalier, seigneur de } \\
\text { Fléville, conseiller du duc et capitaine d'Epinal, a repris de lui ce qu'il tient à } \\
\text { Germiny et qu'il lui a enjoint d'en faire le dénombrement dans les quarante } \\
\text { jours (Nancy, 1530, 25 juin). }\end{array}$ & fol. CXXXIX v ${ }^{\circ}$ \\
\hline 85 & ŹŹ́́ & Le président et les gens des comptes du duché de Bar font savoir qu'ils ont & \\
\hline
\end{tabular}


reçu le dénombrement de ce que tient Nicolas de Lutezelbourg, chevalier, seigneur de Fléville et de Germiny en partie, audit lieu (1536, 30 septembre, 24 août). 